\title{
Gut microbiota was modulated by moxibustion stimulation in rats with irritable bowel syndrome
}

\author{
Xiaomei Wang ${ }^{1,2^{*}+}$, Qin $\mathrm{Qi}^{3 \dagger}$, Yuanyuan Wang ${ }^{3 \dagger}$, Huangan $\mathrm{Wu}^{1,2^{*}}$, Xiaoming Jin ${ }^{4}$, Huan Yao ${ }^{5}$, Duiyin Jin ${ }^{3}$,
} Yanan Liu ${ }^{3}$ and Cun Wang ${ }^{3}$

\begin{abstract}
Background: The pathogenesis of irritable bowel syndrome (IBS) is closely related to intestinal dysbacteriosis and can be controlled by moxibustion treatment. However, the mechanism underlying the therapeutic value of moxibustion in IBS treatment remains unknown.

Methods: An IBS rat model was established by colorectal distention (CRD) stimulus and mustard oil clyster. Sixty-five male rats were randomly divided into six groups: normal, IBS model, moxibustion, electroacupuncture (EA), Bifid-triple Viable Capsule (BTVC) and Pinaverium Bromide (PB) groups. The moxibustion group was treated with mild moxibustion at the bilateral Tianshu (ST25) and Shangjuxu (ST37) for $10 \mathrm{~min} /$ day for 7 days, the EA group was given EA at ST25 and ST37 once daily for 7 days, while the BTVC group and PB groups received Bifid-triple Viable Capsule and Pinaverium Bromide solution (at the proportion of 1:0.018) respectively by gavage once daily for 7 days. After the treatment, abdominal withdrawal reflex (AWR) scores were determined based on CRD stimulus, gut microbiota profiling was conducted by 165 rRNA high-throughput sequencing.
\end{abstract}

Results: Irritable bowel syndrome model rats had significantly increased AWR scores at all intensities $(20,40,60$ and $80 \mathrm{mmHg}$ ) compared with the normal group. Moxibustion treatment significantly reduced AWR scores compared with the IBS model group at all intensities. Across all groups the most abundant phyla were Bacteroidetes and Firmicutes followed by Proteobacteria and Candidatus Saccharibacteria. At genus level IBS model rats had a higher abundance of Prevotella, Bacteroides and Clostridium XI and a lower abundance of Lactobacillus and Clostridium XIVa compared with normal rats. These changes in microbiota profiles could however be reversed by moxibustion treatment. Alpha diversity was decreased in IBS model rats compared with normal rats, yet significantly increased in moxibustion- and PB-treated rats compared with IBS rats.

Conclusion: Our findings suggest that moxibustion treats IBS by modulating the gut microbiota.

Keywords: Irritable bowel syndrome, Moxibustion, Gut microbiota, 165 rRNA

\footnotetext{
*Correspondence: wxm123@vip.sina.com; wuhuangan@126.com

${ }^{\dagger}$ Xiaomei Wang, Qin Qi and Yuanyuan Wang contributed equally to this

work

1 Shanghai Research Institute of Acupuncture and Meridian, Shanghai University of Traditional Chinese Medicine, 650 South Wanping Road, Xuhui District, Shanghai 200030, China

Full list of author information is available at the end of the article
} 


\section{Background}

Irritable bowel syndrome (IBS) is one of the most common gastrointestinal disorders, affecting $10-20 \%$ of the population worldwide $[1,2]$. IBS is characterized by chronic (continuous or intermittent) abdominal pain, bloating, changes in bowel habit and/or stool property. IBS has a multifactorial etiology that may include colonic dysmotility [3], visceral hypersensitivity [4], brain-gut interactions [5], genetic factors [6], post-infectious lowgrade inflammation [7] and altered gut microbiota [8].

Along with the development of microecology theories, the role of the gut microbiota in IBS has been paid increasing attention in recent years. There are trillions of bacteria in the human gut that have co-evolved with us [9]. The predominant phyla in the human gut are Firmicutes and Bacteroidetes, followed by Proteobacteria, Actinobacteria, Fusobacteria and Verrucomicrobia [10]. The human gut is home to a rich variety of microbes. Accordingly, the human intestinal track, particularly the colon, is equipped with sophisticated regulatory mechanisms that facilitate intestinal balance despite complex interaction with the gut microbiota. However, once intestinal balance is disturbed chronic diseases including inflammatory bowel disease [11], allergic diseases [12], obesity [13], colorectal cancer [14] among others [15] may ensue. IBS is closely linked to alterations in gut microbiota composition [16], which can lead to increased permeability of the intestinal mucosal barrier and modulation of cytokine secretion, thus playing a significant role in the pathophysiology of IBS.

Patients with IBS generally have a reduced quality of life [17], underscoring the importance of addressing these symptoms. The treatment of IBS ranges from pharmaceutical to psychological intervention [18]. However, long-term use of currently prescribed therapeutics, such as 5-hydroxytryptamine receptor $\left(5-\mathrm{HT}_{3}\right)$ antagonists, although partly effective, does have several side effects. Psychological treatment does not have any side effects but it is difficult to apply effectively long-term. Moxibustion is a traditional Chinese therapy used to improve general health and treat chronic conditions by stimulating specific points with heat generated by burning herbal preparations containing dried mugwort leaves [19]. Both temperature-related mechanisms and nontemperaturerelated mechanisms likely underlie the effects of moxibustion. The latter includes smoke, herbs, and far infrared effects [20]. Growing evidence supports moxibustion as a safe and effective treatment for IBS [21]. Interestingly, moxibustion has been shown to regulate intestinal microbiota [22]. However, few studies have explored the effect of moxibustion on the intestinal microbiota. We therefore used high-throughput sequencing to determine changes in intestinal microbial community structure in an IBS rat model with or without moxibustion treatment. Our results provide new leads regarding the pathogenesis and treatment of IBS.

\section{Materials and methods}

The Minimum Standards of Reporting Checklist (Additional file 1) contains details of the experimental design, and statistics, and resources used in this study.

\section{Experimental animals}

A total of 65 specific-pathogen free 8-day-old male Sprague-Dawley rats were provided by the Department of Laboratory Animal Science of Shanghai University of Traditional Chinese Medicine. The animals were raised under standard conditions at $25 \pm 1{ }^{\circ} \mathrm{C}$ with a relative humidity of $50-70 \%$ and $12 \mathrm{~h}$ light/dark cycle. The rats did not separate from their mother until they were 4 weeks old. All rats were randomly divided into six groups: normal $(\mathrm{n}=11)$, model $(\mathrm{n}=11)$, moxibustion $(\mathrm{n}=11)$, electroacupuncture $(\mathrm{EA}, \mathrm{n}=10)$, Bifid-triple Viable Capsule (BTVC, $\mathrm{n}=11$ ) and Pinaverium Bromide $(\mathrm{PB}, \mathrm{n}=11)$. All animal work was performed according to the protocols approved by the University Animal Care and Use Committee of Shanghai University of Traditional Chinese Medicine [IACUC protocol number: SYXK (Shanghai) 2009-0082] to reduce pain and to avoid damage. All efforts were made to minimize animal suffering. During establishing IBS model rats, operations should be slow and soft to avoid causing pain and distress. After the procedure, the animals were monitored until fully free to move and eat. For animal therapy, be gentle when catching animals, and take appropriate treatment after the animals calm down. At the end of the experiment, animals received a lethal dose of pentobarbital sodium to minimize animal suffering.

\section{Establishment of the IBS rat model}

The IBS rat model was established by colorectal distention (CRD) through mechanical and chemical stimulus as previously described [23]. An inflatable balloon (Shanghai Dinghuang Industrial Co., Ltd. China) was slowly inserted rectally about $2 \mathrm{~cm}$ into the descending colon of rats. The balloon was distended with $0.5 \mathrm{ml}$ of air, for $1 \mathrm{~min}$ and then repeated after $30 \mathrm{~min}$. The same distention was performed for 14 consecutive days between the age of 8 and 21 days. After 4 weeks rest, mustard oil (0.2 ml, 4\%, Shanghai Zhixin Chemical Co., Ltd. China.) was injected into the descending colon from the anus once a day for 14 days.

\section{Treatment groups}

After successful establishment of the model, rats in the moxibustion group, EA group, BTVC and PB group 
received their relevant treatments. For the moxibustion group, the ignited moxa stick $(0.5 \mathrm{~cm}$ in diameter) (Nanyang Hanyi Moxa Co., Ltd. China) was placed $2 \mathrm{~cm}$ above the bilateral Tianshu (ST25) and Shangjuxu (ST37) acupoints for $10 \mathrm{~min} /$ day for 7 days. ST25 is located bilaterally $5 \mathrm{~mm}$ lateral to the intersection between the upper $2 / 3$ and the lower $1 / 3$, in the line between the xiphoid process and the pubic symphysis upper border and ST37 is $5 \mathrm{~mm}$ lateral to the anterior tubercle of the tibia and $15 \mathrm{~mm}$ below the knee joint [24].

The EA group was given EA at the bilateral Tianshu and Shangjuxu acupoints with Han's Acupoint Nerve Stimulator (Beijing Huawei Industrial Development Corporation. China. LH402A) for sparse-dense waves (frequency of sparse wave: $2 \mathrm{~Hz}$, frequency of dense wave: $10 \mathrm{~Hz}$, intensity: $4 \mathrm{~mA}$ ) for $20 \mathrm{~min}$, once daily for 7 days. The BTVC and PB groups received Bifid-triple Viable Capsule (Inner Mongolia Shuangqi Pharmaceutical Co., Ltd. China. Lot number: S19980004) and Pinaverium Bromide (Abbott Healthcare SAS. France. Lot number: H20120127), respectively by gavage, once daily for 7 days. The BTVC and PB solutions were prepared as specified for a weight ratio of 1:0.018 for an adult $(70 \mathrm{~kg})$ and a rat $(200 \mathrm{~g})$. Prepare the required dose of suspension with drinking water. The BTVC solution concentration was $2 \mathrm{mg} / \mathrm{ml}$ with a daily dose of $20 \mathrm{mg} / \mathrm{kg}$. The PB solution concentration was $5 \mathrm{mg} / \mathrm{ml}$ with a daily dose of $50 \mathrm{mg} /$ $\mathrm{kg}$. The normal and model groups did not receive any treatment. Two rats were died in BTVC group during the treatment by gavage.

\section{Abdominal withdrawal reflex (AWR) scores}

Abdominal withdrawal reflex scores were calculated to assess colon sensitivity to CRD after treatments according to $\mathrm{Al}$-Chaer et al. [23]. Distention was produced by inflating a balloon inside the descending colon through the anus; the inflation balloon had four pressure grades: 20, 40, 60 and $80 \mathrm{mmHg}$. Each CRD lasted about $20 \mathrm{~s}$ and was repeated three times. AWR scores were produced blindly with no subjective judgment. The mean score for each rat was used for downstream analysis. The detailed grading rules on AWR scores are as follows: (0) no behavioral response to CRD; (1) occasional head movement at the onset of the stimulus; (2) mild abdominal muscle contraction but no lifting; (3) strong abdominal muscle contraction and the abdomen but not pelvic structure being lifted off the platform; (4) body arching and lifting of pelvic structures off the platform.

\section{Preparation of fecal and colon tissue samples}

After calculating the AWR scores, rats were weighed and injected with $2 \%$ pentobarbital sodium (Sigma. USA. P3761). The colon samples $(5 \mathrm{~cm}$ above the anus, $3 \mathrm{~cm}$ in length) were rapidly collected from the descending colon, $5 \mathrm{~g}$ fecal matter was collected and stored at $-80{ }^{\circ} \mathrm{C}$ for $16 \mathrm{~S}$ rRNA sequencing. Then, colon samples were fixed in $10 \%$ paraformaldehyde for hematoxylin-eosin staining for histopathological observation.

\section{Fecal DNA extraction}

Bacterial genomic DNA was extracted from all fecal samples using the QIAamp DNAMini Kit (QIAGEN, Germany) according to the manufacturer's instructions. First, $100 \mathrm{mg}$ fecal sample and $1.4 \mathrm{ml}$ buffer ASL were added to a $2 \mathrm{ml}$ tube. Next, $20 \mu \mathrm{l}$ proteinase $\mathrm{K}$ was added to the tube and mixed well before incubation at $56^{\circ} \mathrm{C}$ until the sample was fully dissolved. Next $200 \mu \mathrm{l}$ buffer AL was added to the tube, mixed thoroughly, followed by incubation at $70{ }^{\circ} \mathrm{C}$ for $10 \mathrm{~min}$. Subsequently, $200 \mu \mathrm{l}$ ethanol (96\%) was added to the mixture, which was then loaded onto the QIAamp Mini spin column and centrifuged at $8000 \mathrm{rpm}$ for $1 \mathrm{~min}$. The column material was washed with $500 \mu \mathrm{l}$ buffer AW1 and centrifuged at $8000 \mathrm{rpm}$ for $1 \mathrm{~min}$, then with $500 \mu \mathrm{l}$ buffer AW2 and centrifuged at $14,000 \mathrm{rpm}$ for $3 \mathrm{~min}$. Finally, the DNA was eluted in 100 $\mu \mathrm{l}$ of AE elution buffer. DNA integrity and fragment size range was assessed by agarose gel electrophoresis, and DNA concentrations were measured using a NanoDrop ND-2000 spectrophotometer (Thermo Fisher Scientific, USA).

\section{Illumina MiSeq sequencing}

The V3-V4 region of the bacterial 16S rRNA gene was amplified by polymerase chain reaction (PCR) using universal bacterial primers $341 \mathrm{~F}$ and 806R [25]. Pooled amplicons were sequenced on a 300 PE Illumina MiSeq. Demultiplexed reads were quality filtered based on sequence length and quality as previously described [26]. Operational taxonomic units (OTUs) were clustered at 97\% similarity, and chimeric sequences were removed using UCHIME [27]. Finally, taxonomic assignment of representative sequences was preformed using the Ribosomal Database Project (RDP) MultiClassifier tool [28].

\section{Statistical analyses}

AWR scores was analysed using SPSS21.0 software, and data were expressed as mean \pm SD (Standard \pm Deviation) for normally distributed data and as $M\left(Q_{25}-Q_{75}\right)$ for non-normally distributed data. One-way analysis of variance (ANOVA) was performed for normally distributed data and a non-parametric test (Kruskal-Wallis $H$ test.) was used for non-normally distributed data.

Bioinformatic analyses were performed using $R$ 3.2.3 (http://cran.r-project.org). Differences in relative abundance between groups were assessed using the Kruskal-Wallis test. Alpha diversity was calculated using 
Simpson's diversity index. Beta diversity was determined by analysis of similarities (ANOSIM) using unweighted UniFrac as distance metric. In addition, OTUs that are differentially abundant were determined using Linear discriminant analysis effect size (LefSE). Results were deemed significant if $P<0.05$.

\section{Results}

Abdominal withdrawal reflex (AWR) scores

As shown in Fig. 1, AWR scores were significantly increased in IBS model rats compared with normal rats at all four CRD pressures $(P<0.01)$. AWR scores were however significantly reduced in IBS model rats following treatment with moxibustion at $20(P<0.05), 40,60$ and $80 \mathrm{mmHg}(P<0.01)$. AWR scores of the EA, BTVC and $\mathrm{PB}$ groups also were significantly lower than in IBS model group (EA group: $20 \mathrm{mmHg} P<0.05,40,60$ and $80 \mathrm{mmHg}, P<0.01$; BTVC group: $40 \mathrm{mmHg}, P<0.01$; $\mathrm{PB}$ group: 20, 40 and $60 \mathrm{mmHg}, P<0.05,80 \mathrm{mmHg}, P<0.01$ ). These results suggest that moxibustion treatment could effectively decrease visceral hypersensitivity as EA, BTVC and PB.

\section{Histological analysis}

As shown in Fig. 2, there were no significant differences in histological features between groups. The colonic tissue structure was normal in all groups, and the colonic mucosa epithelium was complete and had regularly arranged glands. There was no congestion, edema, ulcers, inflammatory cell infiltration or other pathological changes in any of the groups.

\section{Gut microbial composition}

A total of 3,759,276 high quality raw sequences were obtained using the MiSeq platform (Illumina, San Diego, CA, USA), and 2,802,729 filtered reads were retained after

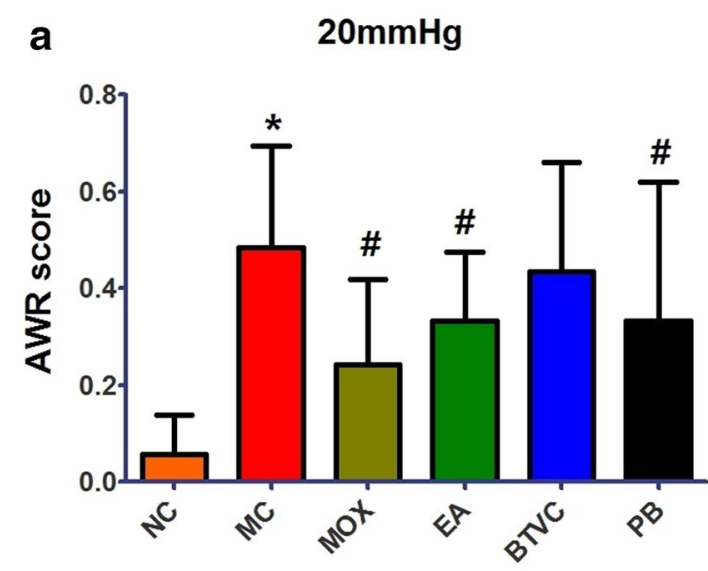

C

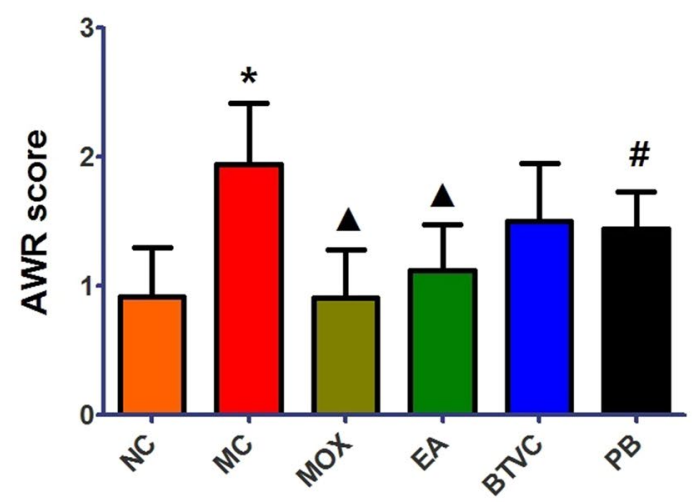

b $40 \mathrm{mmHg}$

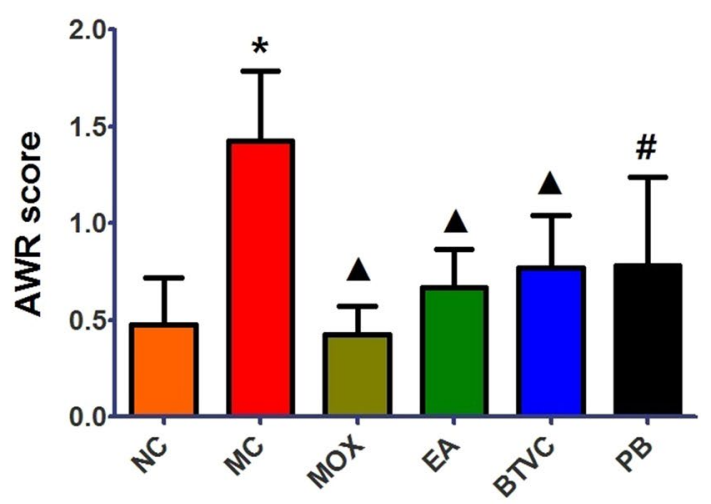

d $80 \mathrm{mmHg}$

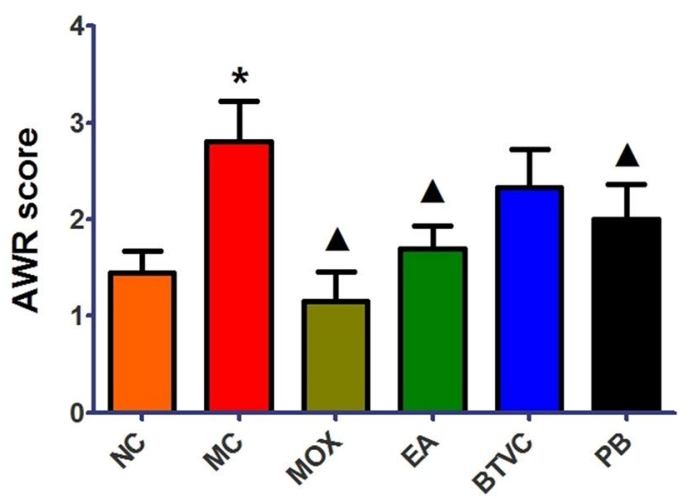

Fig. 1 Abdominal withdrawal reflex (AWR) scores under different distention pressure (a $20 \mathrm{mmHg}$, b 40 mmHg, c 60 mmHg, d $80 \mathrm{mmHg}$ ) in different groups. NC: normal group; MC: IBS model group; MOX: moxibustion group; EA: electroacupuncture group; BTVC: Bifid-triple Viable Capsule group; PB: Pinaverium Bromide group. Data are presented as Median, $\mathrm{Q}_{25}-\mathrm{Q}_{75}$ ( $\mathrm{n}=7$ per group). ${ }^{*} P<0.01$, versus normal group; ${ }^{\sharp} P<0.05, \boldsymbol{\Delta} P<0.01$, versus model group 


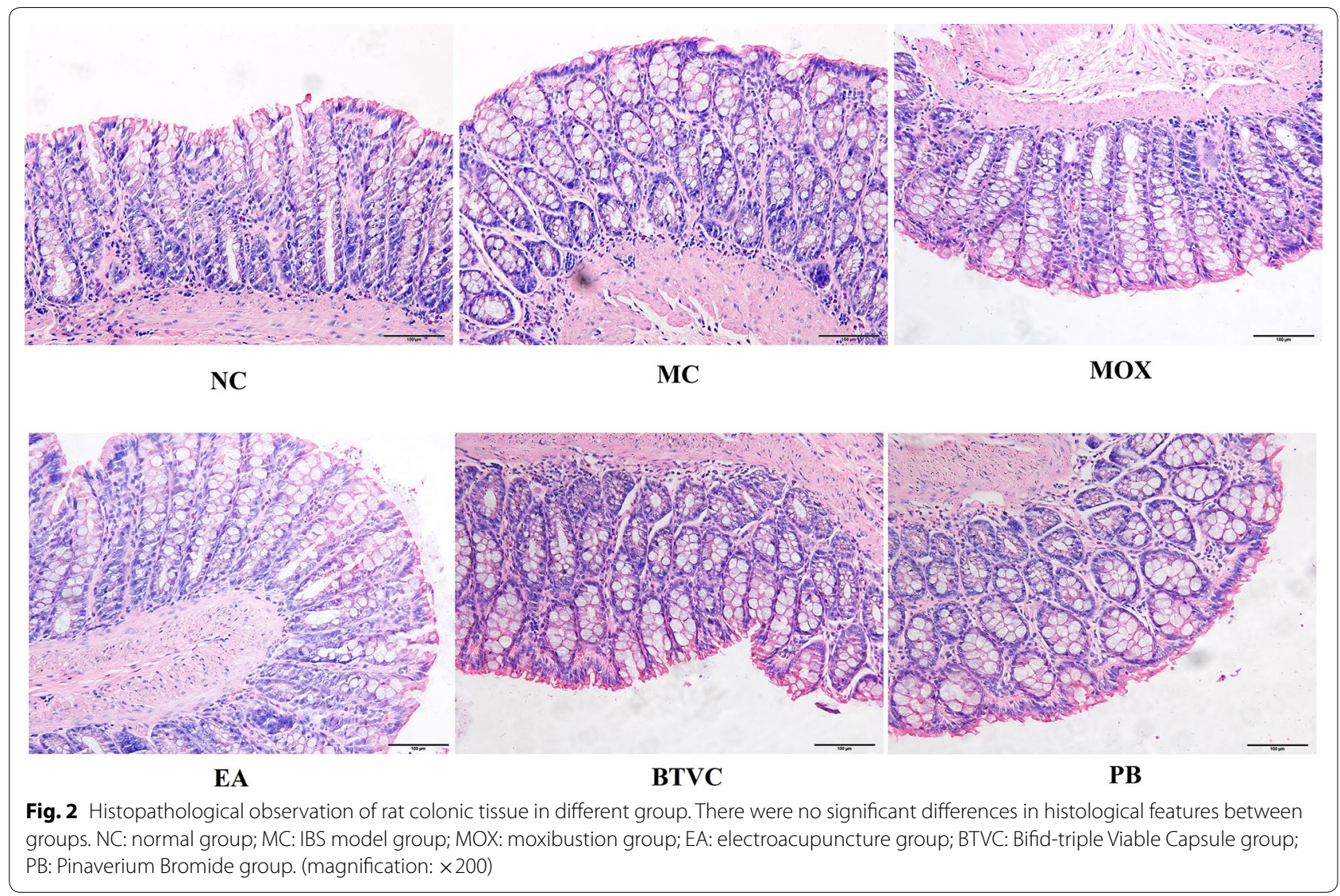

splicing and quality control with an average of 44,487 reads per sample (ranging from 37,081 to 54,506 reads). Reads were then clustered into OTUs at $97 \%$ similarity resulting in 1361 OTUs, which were used for further taxa diversity analysis. In terms of microbial composition, the major phyla present across all groups were Bacteroidetes and Firmicutes, followed by Proteobacteria and Candidatus Saccharibacteria (Fig. 3a). At class level, Bacteroidia and Clostridia were the dominant taxa across all groups, followed by Bacilli and Alphaproteobacteria (Fig. 3b).

\section{Comparison of gut microbial composition between normal and IBS model rats}

At phylum level, both normal and IBS model rats had fecal samples dominated by the phyla Bacteroidetes and Firmicutes. However, the relative abundance of Bacteroidetes and Firmicutes varied significantly between groups.
Compared with the normal group the model group had a higher relative abundance of Bacteroidetes and a lower relative abundance of Firmicutes (Fig. 4a). At genus level, the IBS model group had a higher relative abundance of the genera Prevotella, Bacteroides, Barnesiella, Paraprevotella, Clostridium XI and Sphingomonas compared with normal samples, and a lower relative abundance of Lactobacillus, Clostridium XIVa and Oscillibacter (Fig. 4b).

\section{Comparison of gut microbial composition between IBS model rats with and without moxibustion treatment}

Treatment of IBS model rats with moxibustion led to a fecal microbial profile closer to that of normal rats, with decreased levels of Bacteroidetes and increased levels of Firmicutes following treatment (Fig. 4a). At genus level Prevotella, Bacteroides and Clostridium XI were

\footnotetext{
(See figure on next page.)

Fig. 3 Comparison of overall community structure at phylum and class level by treatment group. a At phylum level, Bacteroidetes and Firmicutes were the dominant taxa across all groups, followed by Proteobacteria and Candidatus Saccharibacteria; $\mathbf{b}$ at class level, Bacteroidia and Clostridia were the dominant taxa across all groups, followed by Bacilli and Alphaproteobacteria. NC: normal group; MC: IBS model group; MOX: moxibustion group; EA: electroacupuncture group; BTVC: Bifid-triple Viable Capsule group; PB: Pinaverium Bromide group
} 


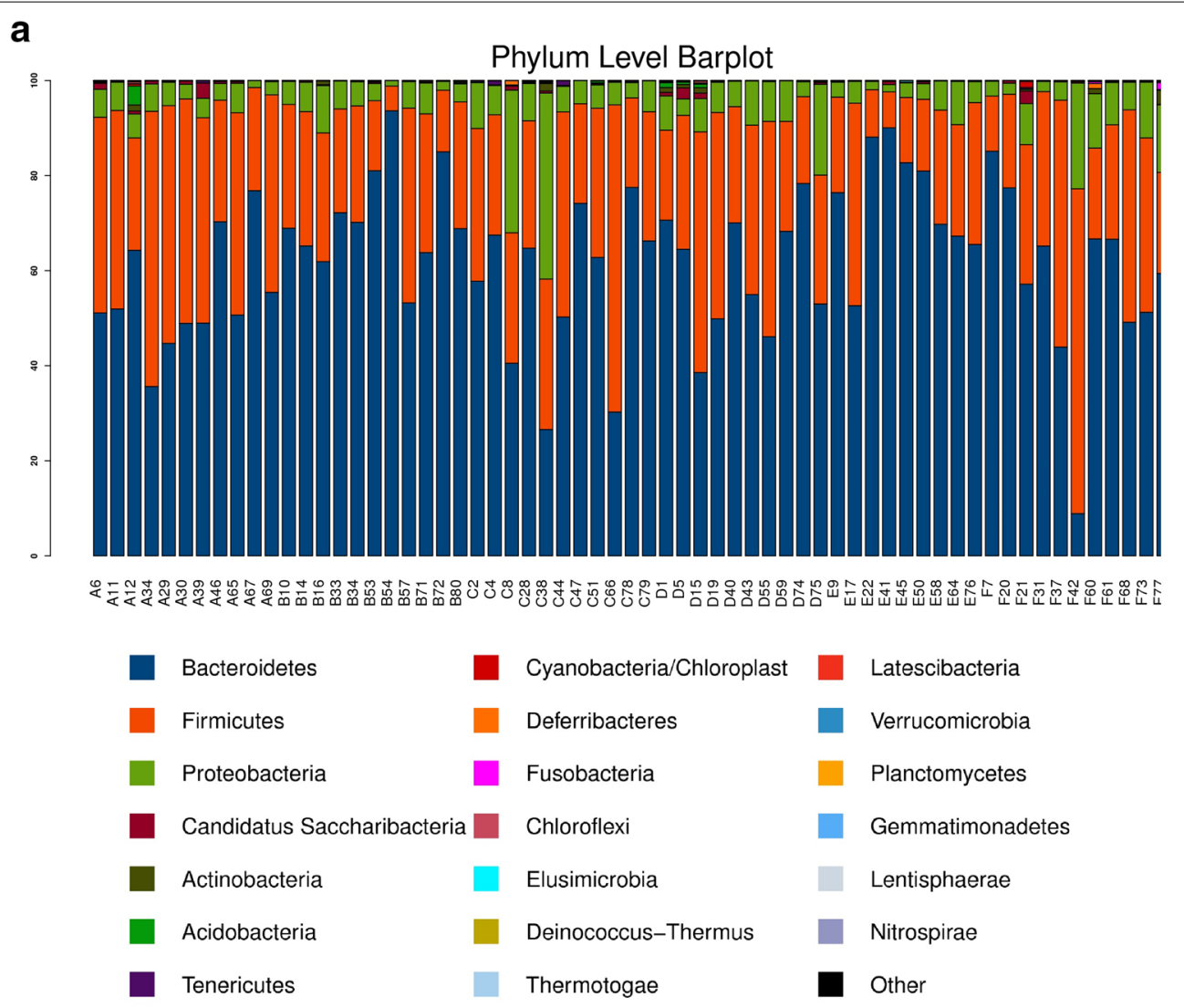

b

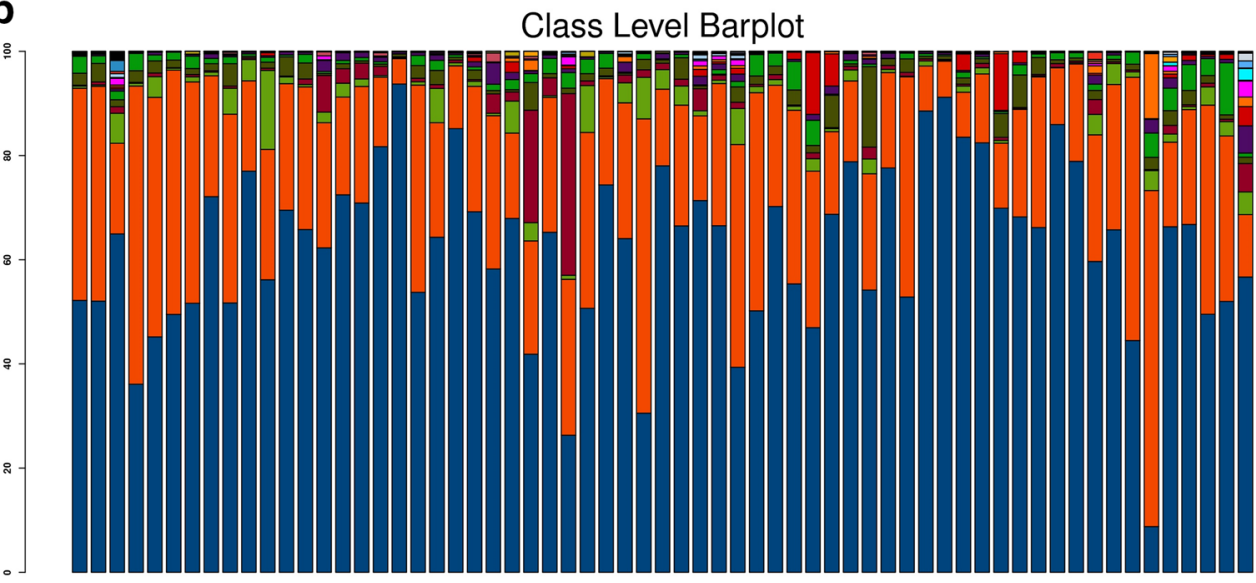

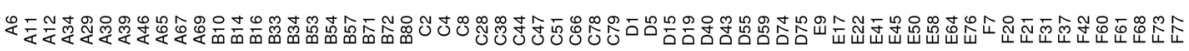
- Bacteroidia
- Negativicutes
Chloroplast
- Clostridia
- Gammaproteobacteria
Acidobacteria_Gp16
Bacilli
Actinobacteria
Deferribacteres
A: NC group
- Alphaproteobacteria
- Erysipelotrichia
- Flavobacteriia
B: MC group
- Deltaproteobacteria
- Sphingobacteriia
Fusobacteriia
$C$ : MOX group
Epsilonproteobacteria
Mollicutes
Thermomicrobia
D: EA group
¿ Betaproteobacteria
Acidobacteria_Gp6
Other
E: BTVC group
F: PB group 

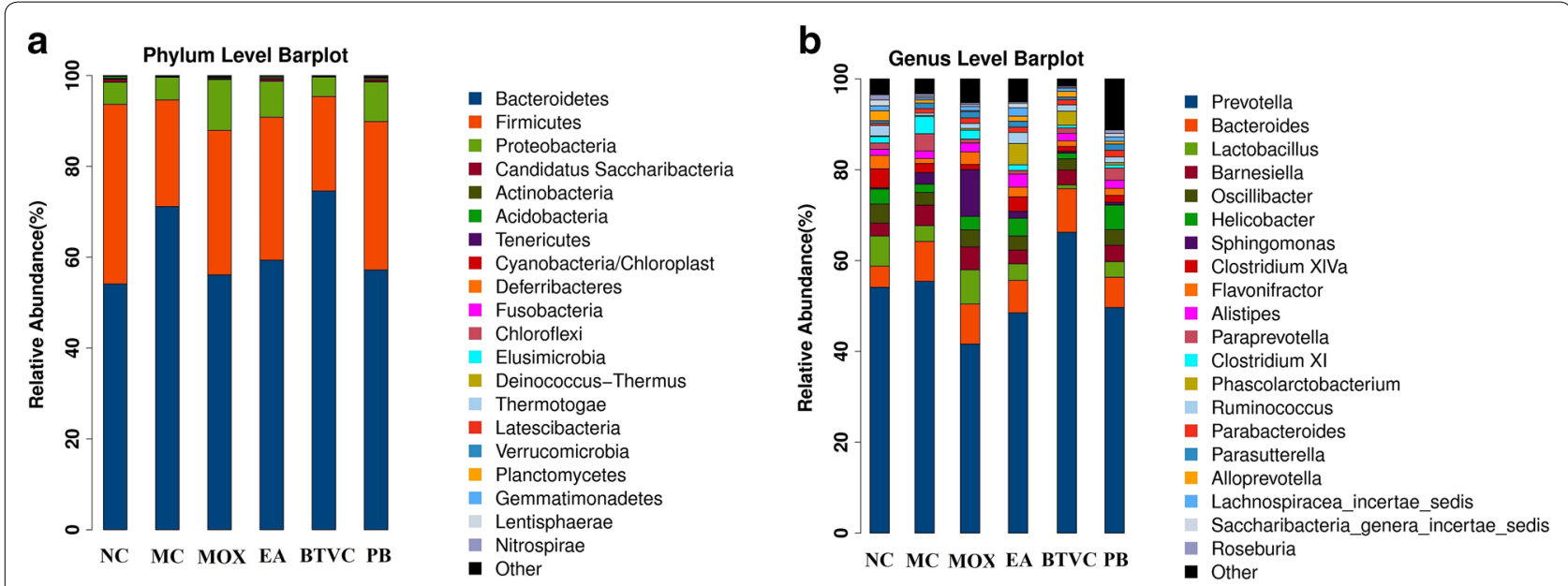

Fig. 4 Microbiota comparison at phylum and genus level. a At phylum level, the relative abundance levels of Bacteroidetes was increased, whereas Firmicutes was decreased in the IBS model group compared with the normal group. These changes in microbiota profiles could be reversed by moxibustion, electroacupuncture, and Pinaverium Bromide treatment. b At genus level, IBS rats had decreased Lactobacillus and increased Prevotella and Bacteroides compared with the normal group. After treatment, Lactobacillus was increased and Prevotella was reduced in the moxibustion, electroacupuncture and Pinaverium Bromide groups. NC: normal group; MC: IBS model group; MOX: moxibustion group; EA: electroacupuncture group; BTVC: Bifid-triple Viable Capsule group; PB: Pinaverium Bromide group

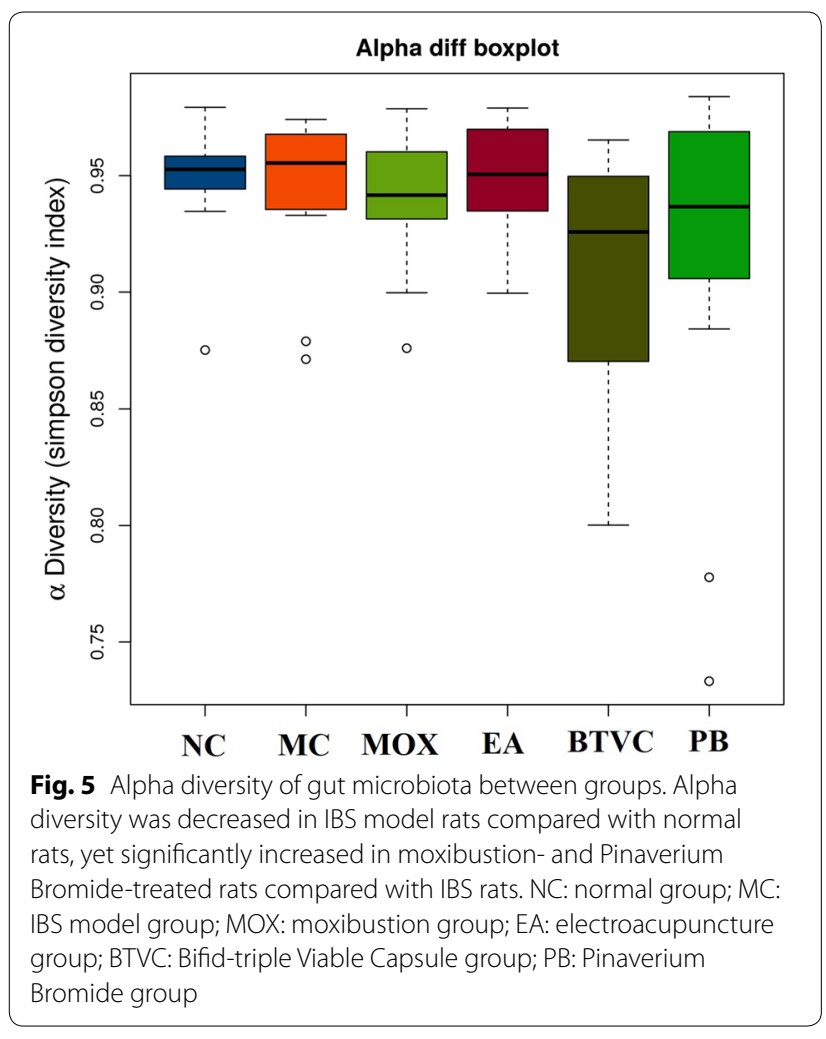

decreased in moxibustion-treated IBS rats while Lactobacillus and Clostridium XIVa were increased in moxibustion-treated IBS rats (Fig. 4b). The relative abundance values were presented in Additional file 2: Table S1.
Richness and diversity of gut bacterial communities

Alpha diversity, as measured by Simpson's diversity index (Fig. 5), was significantly decreased in model compared with normal rats $(P=0.01)$. However, alpha diversity was increased following moxibustion treatment $(P=0.015)$. The EA, BTVC and PB groups also had higher alpha diversity than the model group, which suggests that all these treatments increase gut microbial diversity. With respect to beta diversity, principal coordinates analysis (PCoA) demonstrated significant differences between normal and model groups on the second axis (Fig. 6), suggesting that disease may be the factor influencing microbial community composition. However, the first and second principal coordinates only accounted for $15.04 \%$ and $8.93 \%$ of the total variations respectively, indicating that there are other factors affecting the IBS rats microbial community or more-refined analysis needed. The microbial community composition from EA group was more similar to $\mathrm{PB}$ group, and moxibustion was more similar to BTVC on the first axis.

\section{Fecal biomarkers of IBS and different treatments}

Linear discriminant analysis effect size (LEfSe), a biomarker discovery tool for high dimensional data, was used to determine which OTUs were differentially abundant between normal and model samples and model and different treatments samples and hence potential biomarkers of IBS and different treatments (Fig. 7a, b). A total of 37 OTUs at different taxonomic levels were differentially abundant $(\mathrm{P}<0.05)$ between 


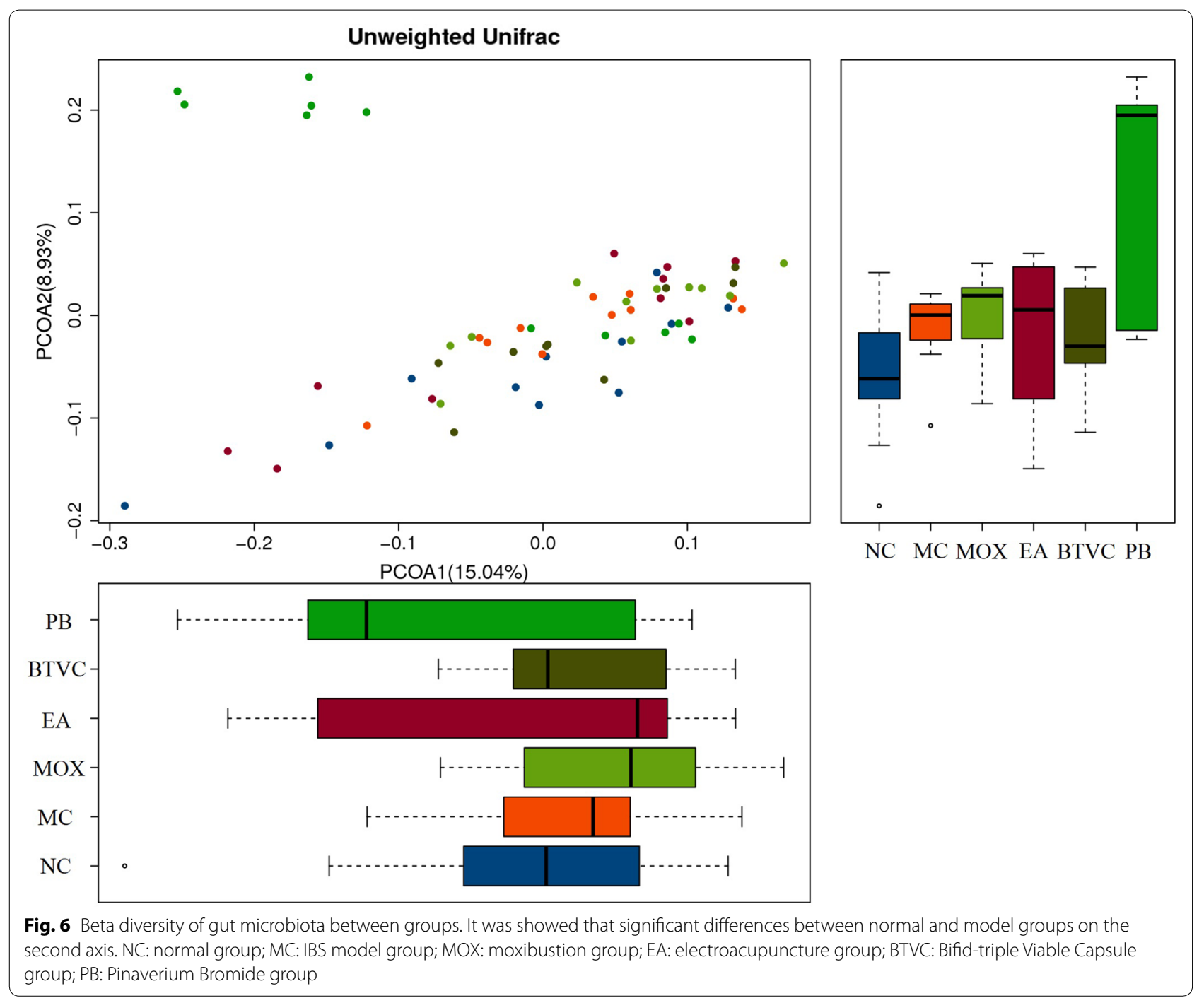

normal and model samples. At phylum level, the relative abundance of Bacteroidetes and Firmicutes were increased and decreased in IBS model rats, respectively. At class level Bacterodia, Alphaproteobacteria, Betaproteobacteria and Erysipelotrichia were highly enriched in IBS model rats, while Epsilonproteobacteria and Clostridia were enriched in normal rats. At order level, Bacteroidales, Sphingomonadales, Burkholderiales and Erysipelotrichales were significantly enriched in the IBS model rats. At family level Porphyromonadaceae, Peptostreptococcaceae, Sphingomonadaceae, Sutterellaceae, Burkholderiales and Erysipelotrichaceae were enriched in IBS model rats, while Helicobacteraceae, Ruminococcaceae and Lachnospiraceae were enriched in normal rats. Similarly, the genera Advenella, Psychrobacter, Clostridium XI, Sphingomonas, Parasutterella and Aquabacterium were significantly more abundant in IBS model rats, whereas normal rats were enriched with Clostridium IV, Butyricicoccus, Saccharibacteria, Helicobacter, Ruminococcus, Clostridium XIVa, and Faecalibacterium. These results are represented by heatmap analyses on a per-sample basis in Fig. 7c.

A total of 14 OTUs at different taxonomic levels were differentially abundant $(\mathrm{P}<0.05)$ between $\mathrm{MC}$ and MOX group. Among them, compared with MC group, the relative abundance of Ruminococcaceae, Enterobacteriaceae, Clostridiaceae1, Enterobacteriales, Escherichia Shigella, Clostridiumsensustricto, Butyricicoccus and Enterorhabdus were significant abundant in MOX group which may be the potential biomarkers of the moxibustion to treat UC (Fig. 8a, b). A total of 17 OTUs at different taxonomic levels were differentially abundant $(\mathrm{P}<0.05)$ between $\mathrm{MC}$ and EA group. Similarly, the relative abundance of Negativicutes, Selenomonadales, Gammaproteobacteria, Buttiauxella, Bacillaceae2, Butyricicoccus, Enterobacteriales, Enterobacteriaceae and Virgibacillus were significant 


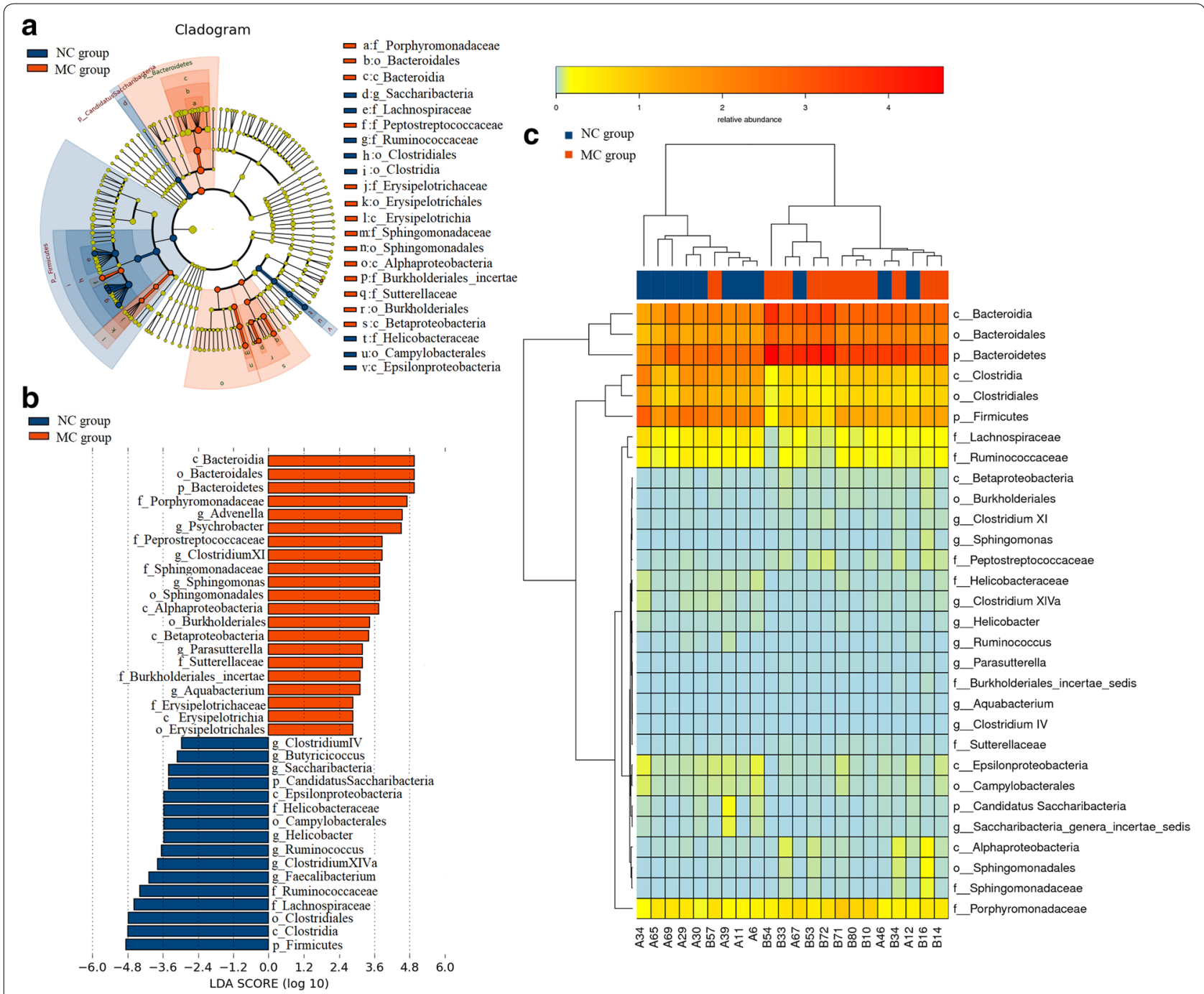

Fig. 7 Differentially abundant OTUs between normal and IBS model rats using LEfSe analysis. a Taxonomic cladogram of differences in the gut microbiota between normal and IBS model groups, blue shows tax enriched in normal group, red IBS model group and yellow non-significant. The diameter of each circle is proportional to the abundance of taxon. $\mathbf{b}$ The most abundant taxa in normal group with blue histogram and IBS model group with red histogram. c Heatmap of OTUs found to be significantly differentially abundant between normal and IBS model rats. NC: normal group; MC: IBS model group

abundant in EA group which may be the potential biomarkers of the electroacupuncture to treat UC (Fig. 8c, d). Interestingly, Butyricicoccus, Enterobacteriales and Enterobacteriaceae were significant abundant both in MOX and EA group then MC group.

A total of 26 OTUs at different taxonomic levels were differentially abundant $(\mathrm{P}<0.05)$ between $\mathrm{MC}$ and BTVC group. Among them, Clostridiales IncertaesedisXI and Parvimonas were significant abundant in BTVC group then MC group (Fig. 9a, b). A total of 81 OTUs at different taxonomic levels were differentially abundant $(\mathrm{P}<0.05)$ between $\mathrm{MC}$ and $\mathrm{PB}$ group. Compared with MC group, there were 69 taxa significant abundant in
PB group which may be the potential biomarkers of the Pinaverium Bromide to treat UC, such as Ruminococcus, Butyricicoccus, Fusobacteria, Deinococcales, Thermotogae, Vibrionales, Epsilonproteobacteria and so on (Fig. 9c, d).

\section{Discussion}

IBS is characterized by several symptoms, including abdominal pain, that can seriously affect quality of life. Visceral hypersensitivity (enhanced intestinal perception) plays a significant role in such abdominal pain and discomfort [4]. In this study, we applied AWR scores to assess visceral hypersensitivity in rats. We found 


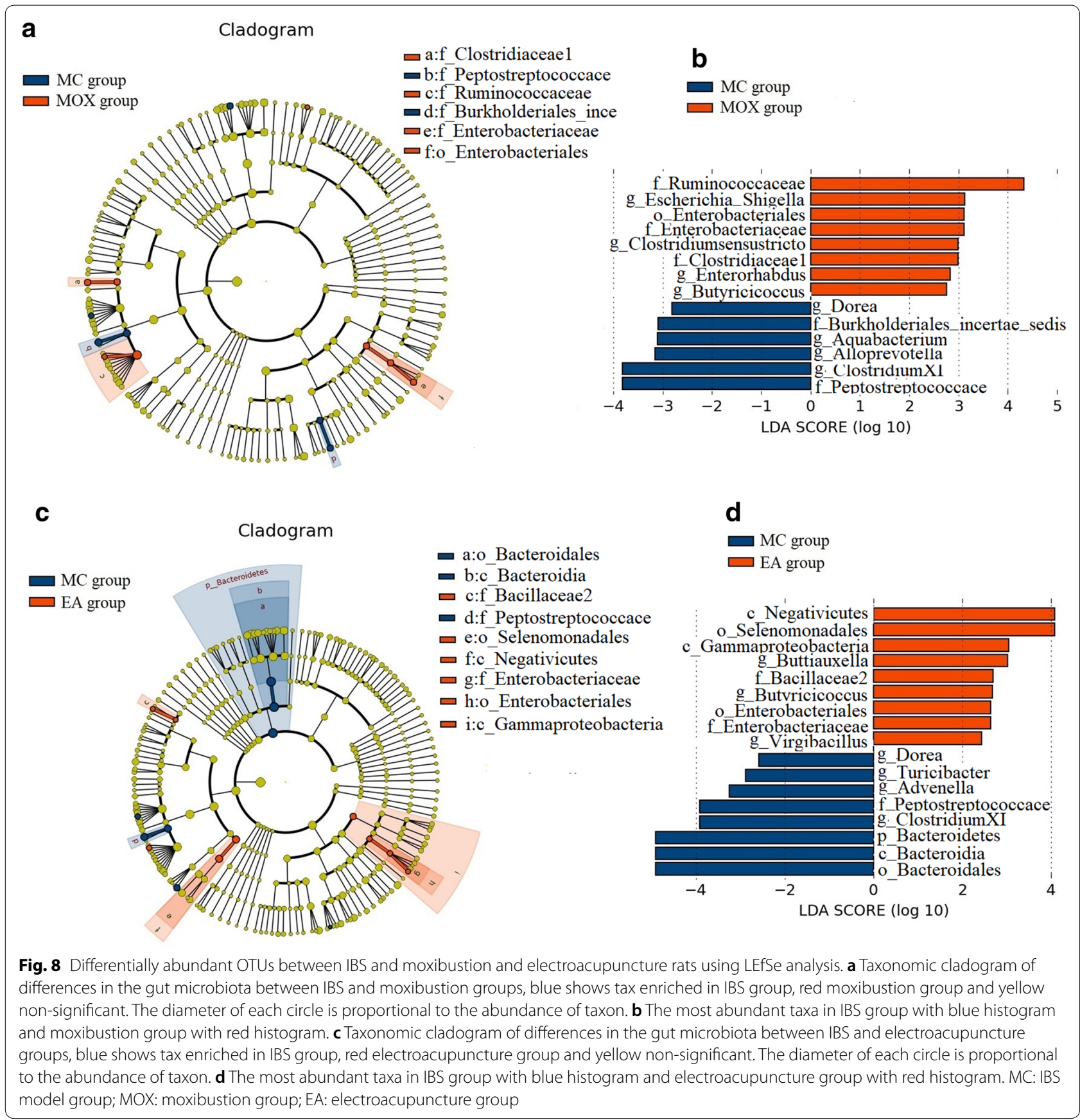

\section{(See figure on next page.)}

Fig. 9 Differentially abundant OTUs between IBS and Bifid-triple Viable Capsule and Pinaverium Bromide rats using LEfSe analysis. a Taxonomic cladogram of differences in the gut microbiota between IBS and Bifid-triple Viable Capsule groups, blue shows tax enriched in IBS group, red Bifid-triple Viable Capsule group and yellow non-significant. The diameter of each circle is proportional to the abundance of taxon. $\mathbf{b}$ The most abundant taxa in IBS group with blue histogram and Bifid-triple Viable Capsule group with red histogram. c Taxonomic cladogram of differences in the gut microbiota between IBS and Pinaverium Bromide groups, blue shows tax enriched in IBS group, red Pinaverium Bromide group and yellow non-significant. The diameter of each circle is proportional to the abundance of taxon. $\mathbf{d}$ The most abundant taxa in IBS group with blue histogram and Pinaverium Bromide group with red histogram. MC: IBS model group; BTVC: Bifid-triple Viable Capsule group; PB: Pinaverium Bromide group 


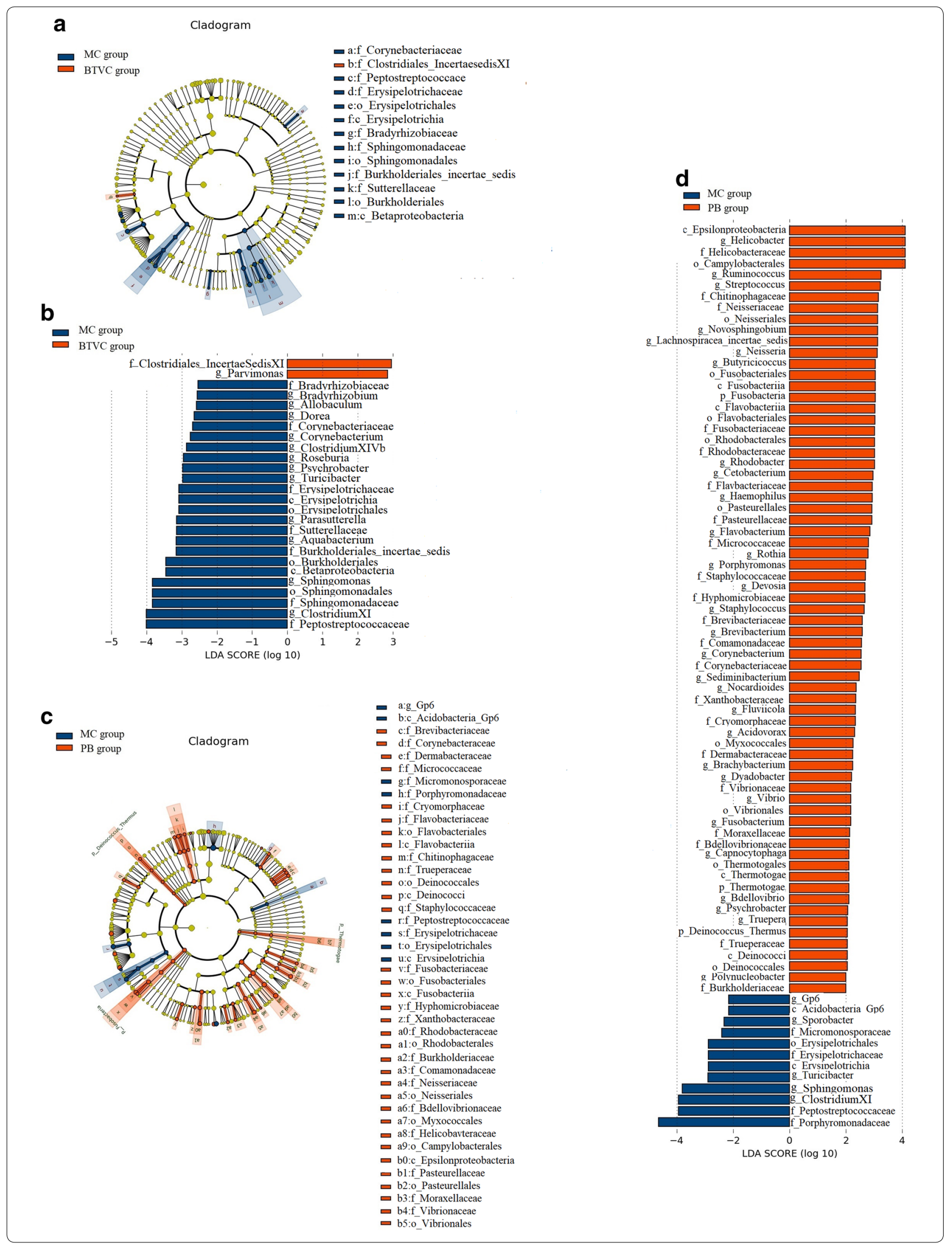


that AWR scores of IBS model rats were significantly increased compared with normal rats. Certain studies have shown that patients with IBS have a higher pain sensitivity and lower pain threshold than normal subjects [29]_our results support these findings. More importantly, AWR scores of IBS rats were significantly decreased in moxibustion, EA, BTVC and PB groups, which demonstrates that moxibustion and EA can effectively alleviated abdominal pain by increasing pain threshold and decreasing visceral hypersensitivity in IBS rats as Pinaverium Bromide and Bifid-triple Viable Capsule. Several studies have reported that electroacupuncture [30], probiotic [31] and Pinaverium Bromide [32] have therapeutic effect for IBS. Our findings indicate that moxibustion may potentially be used as an alternative treatment to Bifid-triple Viable Capsule and Pinaverium Bromide.

The intestinal microbiota profoundly affects human health through various means. Commensal bacteria promote proper functioning of the physical and biochemical barrier against pathogens as well as immune system development [33]. Intestinal bacteria and their metabolic products interact with the host gut mucosal surface thereby shaping the host immune system. Under healthy conditions the host's response to these bacterial signals will result in immune tolerance. Normal intestinal microbiota play a critical role in promoting immune system development, sustaining normal immune function, and preventing infection by pathogens [34]. However, when dysbacteriosis occurs the balance between tolerance towards commensals and immune activation in response to pathogens may be lost, which may lead to a range of diseases.

Tianshu and Shangjuxu acupoints are ancient and classical acupoint combination for intestinal diseases such as diarrhea and abdominal pain [35]. Numerous studies suggest that dysbacteriosis is closely related to the pathophysiology of IBS [36]. Moxibustion has proven benefits in treating IBS [37]. However, ours is the first study to examine the effect of moxibustion on the gut microbiota in IBS. We analyzed changes in gut microbiota between IBS and normal rats and the effect of moxibustion therapy on the gut microbiota.

We found that the intestinal microbial composition of IBS rats differed from that of normal rats. IBS rats had significantly decreased alpha diversity and increased relative abundance of Bacteroidetes, which is consistent with previous reports [38]. Several studies have now reported that IBS patients and IBS model rats have significantly reduced levels of Lactobacillus [39, 40]. Lactobacillus is a major component of the commensal bacterial flora of the human intestinal tract, and is frequently used as a probiotic as it induces the production of large quantities of anti-inflammatory interleukins that improve intestinal barrier function, thus preventing the development of colitis [41]. Several studies have shown that Lactobacillus GG-a specific probiotic strain of Lactobacillus (ATCC 53103)-effectively treats IBS in humans and rats [42-44]. Indeed, in our study, Lactobacillus was decreased in IBS rats, as were Clostridium XIVa and Oscillibacter. Further, Prevotella, Bacteroides and Clostridium XI were increased in IBS model rats. Interestingly however, these IBS-related changes in gut microbiota could be normalized by moxibustion treatment, after which the relative abundance of Lactobacillus and Clostridium XIVa increased, while Prevotella, Bacteroides and Clostridium XI decreased. In addition, moxibustion treatment led to increased gut microbiota diversity, as did the other treatments considered in this study (EA, BTVC, and PB) to varying degrees.

We conducted LEfSe to discover distinctive features at all levels which may be the potential biomarkers of the IBS. Twenty-one features were discovered by LEfSe, and the relative abundance of Bacteroidia, Bacteroidales and Bacteroidetes, which exhibited the top three highest LDA score suggesting that these features may be closely related to IBS. We have also identified some potential markers that may play a therapeutic role in different treatments. It was an interesting finding that Butyricicoccus, Enterobacteriales and Enterobacteriaceae were significant abundant both in MOX and EA group compared to MC group. This suggests that moxibustion and electroacupuncture may have some similar therapeutic targets. Although we have found some potential biomarkers, how to regulate these markers by moxibustion and electroacupuncture still requires further research.

\section{Conclusions}

Our findings suggest that moxibustion treats IBS by modulating the gut microbiota. We demonstrate that moxibustion could potentially be used to regulate gut microbiota imbalances and therefore to treat patients with IBS.

\section{Additional files}

Additional file 1. The Minimum Standards of Reporting Checklist.

Additional file 2: Table S1. Microbiota comparison at phylum, class and genus levels.

\section{Abbreviations}

IBS: irritable bowel syndrome; CRD: colorectal distention; EA: electroacupuncture; BTVC: Bifid-triple Viable Capsule; PB: Pinaverium Bromide; AWR: abdominal withdrawal reflex; $5-\mathrm{HT}_{3}$ : 5-hydroxytryptamine receptor; PCR: polymerase 
chain reaction; OTUs: operational taxonomic units; RDP: Ribosomal Database Project; PCoA: principal coordinates analysis; ANOVA: one-way analysis of variance; ANOSIM: analysis of similarities; LEfSe: Linear discriminant analysis effect size.

\section{Authors' contributions}

WX and WH conceived and designed the study. WX and QQ wrote the main manuscript text. WY, LY and JD performed animal experiments and collected data. JX and YH analyzed data. WC prepared figures and tables. All authors reviewed the manuscript. All authors read and approved the final manuscript.

\section{Author details}

${ }^{1}$ Shanghai Research Institute of Acupuncture and Meridian, Shanghai University of Traditional Chinese Medicine, 650 South Wanping Road, Xuhui District, Shanghai 200030, China. ${ }^{2}$ Key Laboratory of Acupuncture and Immunological Effects, Shanghai University of Traditional Chinese Medicine, Shanghai 200030, China. ${ }^{3}$ Yueyang Clinical Medical College, Shanghai University of Traditional Chinese Medicine, Shanghai 200437, China. ${ }^{4}$ Stark Neurosciences Research Institute \& Department of Anatomy and Cell Biology, Indiana University School of Medicine, Indianapolis, IN 46202, USA. ${ }^{5}$ Department of Radiation Oncology, Indiana University School of Medicine, Indianapolis, IN 46202, USA.

\section{Acknowledgements}

Sequencing data analysis was supported by Realbio Genomics Institute.

\section{Competing interests}

The authors declare that they have no competing interests.

\section{Availability of data and materials}

The datasets used during the current study are available from the corresponding author on reasonable request.

\section{Consent for publication}

Not applicable.

\section{Ethics approval and consent to participate}

The study was review and approved by the Ethics Committee of Yueyang Hospital of Integrated Traditional Chinese and Western Medicine, Shanghai University of Traditional Chinese Medicine.

\section{Funding}

This work was supported by the Natural Science Foundation of Shanghai (Grant Number 14ZR1438700), the National Basic Research Programme of China (973 programme, Grant Number 2015CB554501) and National Natural Science Foundation of China (Grant Number 81473758).

\section{Publisher's Note}

Springer Nature remains neutral with regard to jurisdictional claims in published maps and institutional affiliations.

Received: 3 October 2018 Accepted: 14 December 2018 Published online: 18 December 2018

\section{References}

1. Canavan C, West J, Card T. The epidemiology of irritable bowel syndrome. Clin Epidemiol. 2014;6:71-80.

2. Zeber-Lubecka N, Kulecka M, Ambrozkiewicz F, Paziewska A, Goryca K, Karczmarski J, et al. Limited prolonged effects of rifaximin treatment on irritable bowel syndrome-related differences in the fecal microbiome and metabolome. Gut Microbes. 2016;7:397-413.

3. Lee OY. Asian motility studies in irritable bowel syndrome. J Neurogastroenterol Motil. 2010;16:120-30.

4. Barshop K, Staller K. New pathways, new targets: visceral hypersensitivity pathogenesis in irritable bowel syndrome. Clin Transl Gastroenterol. 2016;7:e146.

5. Lee YJ, Paek KS. Irritable bowel syndrome: emerging paradigm in pathophysiology. World J Gastroenterol. 2014;20:2456-69.
6. Ratanasirintrawoot S, Israsena N. Stem cells in the intestine: possible roles in pathogenesis of irritable bowel syndrome. J Neurogastroenterol Motil. 2016;22:267-382.

7. Ringel Y, Maharshak N. Intestinal microbiota and immune function in the pathogenesis of irritable bowel syndrome. Am J Physiol Gastrointest Liver Physiol. 2013;305:529-41.

8. Bennet S, Öhman L, Simrén M. Gut microbiota as potential orchestrators of irritable bowel syndrome. Gut Liver. 2015;9:318-31.

9. Qin J, Li R, Raes J, Arumugam M, Burgdorf KS, Manichanh C, et al. A human gut microbial gene catalog established by metagenomic sequencing. Nature. 2010:464:59-65.

10. Khan I, Yasir M, Azhar El, Kumosani T, Barbour EK, Bibi F, et al. Implication of gut microbiota in human health. CNS Neurol Disord Drug Targets. 2014;13:1325-33.

11. Ubeda C, Pamer EG. Antibiotics, microbiota, and immune defense. Trends Immunol. 2012;33:459-66.

12. Huang $Y$, Chen Z. Inflammatory bowel disease related innate immunity and adaptive immunity. Am J Transl Res. 2016;8:2490-7.

13. Penders J, Gerhold K, Thijs C, Zimmermann K, Wahn U, Lau S, et al. New insights into the hygiene hypothesis in allergic diseases: mediation of sibling and birth mode effects by the gut microbiota. Gut Microbes. 2014:5:239-44.

14. Pickett-Blakely O. Obesity and irritable bowel syndrome: a comprehensive review. Gastroenterol Hepatol. 2014;10:411-6.

15. Marchesi JR, Dutilh BE, Hall N, Peters WH, Roelofs R, Boleij A, et al. Towards the human colorectal cancer microbiome. PLOS ONE. 2011;6:e20447.

16. Lopetuso LR, Scaldaferri F, Bruno G, Petito V, Franceschi F, Gasbarrini $A$. The therapeutic management of gut barrier leaking: the emerging role for mucosal barrier protectors. Eur Rev Med Pharmacol Sci. 2015;19:1068-76.

17. Zhu LM, Huang D, Shi LL, Liang LX, Xu T, Chang M, et al. Intestinal symptoms and psychological factors jointly affect quality of life of patients with irritable bowel syndrome with diarrhea. Health Qual Life Outcomes. 2015;13:49.

18. Shi $Y$, Chen $Y H$, Yin XJ, Wang AQ, Chen XK, Lu JH, et al. Electroacupuncture versus moxibustion for irritable bowel syndrome: a randomized, parallelcontrolled trial. Evid Based Complement Altern Med. 2015;2015:361786.

19. Yoon SL, Grundmann O, Koepp L, Farrell L. Management of irritable bowel syndrome (IBS) in adults: conventional and complementary/alternative approaches. Altern Med Rev. 2011;16:134-51.

20. Chiu JH. How does moxibustion possibly work? Evid Based Complement Altern Med. 2013;2013:198584.

21. Huang RJ, Zhao JM, Wu LY, Dou CZ, Liu HR, Weng ZJ, et al. Mechanisms underlying the analgesic effect of moxibustion on visceral pain in irritable bowel syndrome: a review. Evid Based Complement Altern Med. 2014;2014:895914.

22. Wang XM, Lu Y, Wu LY, Yu SG, Zhao BX, Hu HY, et al. Moxibustion inhibits interleukin-12 and tumor necrosis factor alpha and modulates intestinal flora in rat with ulcerative colitis. World J Gastroenterol. 2012;18:6819-28.

23. Al-Chaer ED, Kawasaki M, Pasricha PJ. A new model of chronic visceral hypersensitivity in adult rats induced by colon irritation during postnatal development. Gastroenterology. 2000;119:1276-85.

24. Weng ZJ, Wu LY, Zhou CL, Dou CZ, Shi Y, Liu HR, et al. Effect of electroacupuncture on $\mathrm{P} 2 \mathrm{X} 3$ receptor regulation in the peripheral and central nervous systems of rats with visceral pain caused by irritable bowel syndrome. Purinergic Signal. 2015;11(3):321-9.

25. Takahashi S, Tomita J, Nishioka K, Hisada T, Nishijima M. Development of a prokaryotic universal primer for simultaneous analysis of Bacteria and Archae using next-generation sequencing. PLOS ONE. 2014;9:e105592.

26. Vicente CS, Ozawa S, Hasegawa K. Composition of the cockroach gut microbiome in the presence of parasitic nematodes. Microbes Environ. 2016:31:314-20.

27. Xu J, Chen X, Yu S, Su Y, Zhu W. Effects of early intervention with sodium butyrate on gut microbiota and the expression of inflammatory cytokines in neonatal piglets. PLOS ONE. 2016;11:e0162461.

28. Wang Q, Garrity GM, Tiedje JM, Cole JR. Naive Bayesian classifier for rapid assignment of rRNA sequences into the new bacterial taxonomy. Appl Environ Microbiol. 2007;73:5261-7.

29. Keszthelyi D, Troost FJ, Masclee AA. Irritable bowel syndrome: methods, mechanisms, and pathophysiology. Methods to assess visceral 
hypersensitivity in irritable bowel syndrome. Am J Physiol Gastrointest Liver Physiol. 2012;303:141-54.

30. Zhao JM, Chen L, Zhou CL, Shi Y, Li YW, Shang HX, et al. Comparison of electroacupuncture and moxibustion for relieving visceral hypersensitivity in rats with constipation-predominant irritable bowel syndrome. Evid Based Complement Altern Med. 2016;2016:9410505.

31. Cui S, Hu Y. Multistrain probiotic preparation significantly reduces symptoms of irritable bowel syndrome in a double-blind placebo-controlled study. Int J Clin Exp Med. 2012:5(3):238-44.

32. Hou X, Chen S, Zhang Y, Sha W, Yu X, Elsawah H, et al. Quality of life in patients with irritable bowel syndrome (IBS), assessed using the IBSquality of life (IBS-QOL) measure after 4 and 8 weeks of treatment with mebeverine hydrochloride or pinaverium bromide: results of an international prospective observational cohort study in Poland, Egypt, Mexico and China. Clin Drug Invest. 2014;34(11):783-93.

33. Zeng MY, Cisalpino D, Varadarajan S, Hellman J, Warren HS, Cascalho M, et al. Gut microbiota-induced immunoglobulin $\mathrm{G}$ controls systemic infection by symbiotic bacteria and pathogens. Immunity. 2016;44:647-58.

34. Furusawa Y, Obata Y, Fukuda S, Endo TA, Nakato G, Takahashi D, et al. Commensal microbe-derived butyrate induces the differentiation of colonic regulatory T cells. Nature. 2013;504:446-50.

35. Zhang HS, Wang FC. Application of combination of he-mu points and combination of shu-yuan in syndrome differentiation of zang- and fuorgans. Zhongguo Zhen Jiu. 2006;26(5):378-80.
36. Öhman L, Törnblom H, Simrén M. Crosstalk at the mucosal border: importance of the gut microenvironment in IBS. Nat Rev Gastroenterol Hepatol. 2015:12:36-49.

37. Kondo T, Kawamoto M. Acupuncture and moxibustion for stress-related disorders. Biopsychosoc Med. 2014;8:7.

38. Ponnusamy K, Choi JN, Kim J, Lee SY, Lee CH. Microbial community and metabolomic comparison of irritable bowel syndrome faeces. J Med Microbiol. 2011;60:817-27.

39. Goepp J, Fowler E, McBride T, Landis D. Frequency of abnormal fecal biomarkers in irritable bowel syndrome. Glob Adv Health Med. 2014;3:9-15.

40. Sheikh Sajjadieh MR, Kuznetsova LV, Bojenko VB. Dysbiosis in ukrainian children with irritable bowel syndrome affected by natural radiation. Iran J Pediatr. 2012;22:364-8.

41. Patel R, DuPont HL. New approaches for bacteriotherapy: prebiotics, newgeneration probiotics, and synbiotics. Clin Infect Dis. 2015;60:108-21.

42. Kianifar H, Jafari SA, Kiani M, Ahanchian H, Ghasemi SV, Grover Z, et al. Probiotic for irritable bowel syndrome in pediatric patients: a randomized controlled clinical trial. Electron Physician. 2015;7:1255-60.

43. Shavakhi A, Minakari M, Farzamnia S, Peykar MS, Taghipour G, Tayebi A, et al. The effects of multi-strain probiotic compound on symptoms and quality-of-life in patients with irritablebowel syndrome: a randomized placebo-controlled trial. Adv Biomed Res. 2014;3:140.

44. Tiequn B, Guanqun C, Shuo Z. Therapeutic effects of Lactobacillus in treating irritable bowel syndrome: a meta-analysis. Intern Med. 2015:54:243-9.
Ready to submit your research? Choose BMC and benefit from:

- fast, convenient online submission

- thorough peer review by experienced researchers in your field

- rapid publication on acceptance

- support for research data, including large and complex data types

- gold Open Access which fosters wider collaboration and increased citations

- maximum visibility for your research: over $100 \mathrm{M}$ website views per year

At BMC, research is always in progress.

Learn more biomedcentral.com/submissions 